\title{
White Rabbit Facility
}

\author{
Roberto Ammendola ${ }^{1}$, Aldo Morselli ${ }^{1}$, Gonzalo Rodríguez ${ }^{1}$, , Gaetano Salina ${ }^{1}$, and Valerio Verzi ${ }^{1}$ \\ ${ }^{1}$ Sezione INFN di Tor Vergata, Roma, Italy
}

\begin{abstract}
We describe a White Rabbit Facility for time synchronization we are preparing for the future Cherenkov Telescope Array observatory,
\end{abstract}

\section{Introduction}

Upcoming Gamma-Ray and Cosmic-Ray experiments require relative time calibration of all detector components with (sub-)nanosecond precision. White Rabbit (WR) [1] is a time-deterministic, lowlatency Ethernet-based network that provides nanosecond accuracy and sub-nanosecond precision of clock synchronization for large distributed systems. Its aim is to combine the real-time performance of industrial networks and the flexibility of the Ethernet protocol with the accuracy of dedicated timing systems. White Rabbit is capable of synchronizing more than 1000 nodes, see figure 1, connected by fibers of up to $10 \mathrm{~km}$ in length, with sub-nanosecond accuracy. It uses a robust, prioritized message delivery system. White Rabbit time synchronization packets are routinely flagged as high priority, but other critical Ethernet packets may be priority-flagged as well.

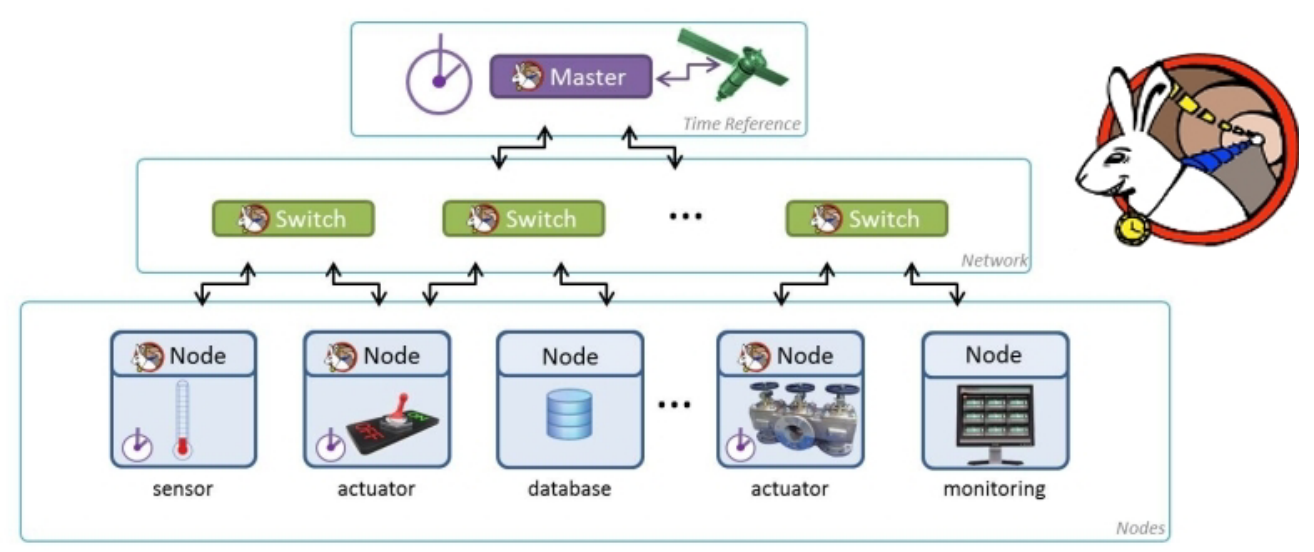

Figure 1: Scheme of White Rabbit network, from http://www.ohwr.org/projects/white-rabbit/wiki

\footnotetext{
ae-mail: grferna@roma2.infn.it
} 
WR is currently being implemented as the next version of the IEEE 1588 standard, the Precision Time Protocol (PTP) and there is a large and active community of White Rabbit users and contributors (CERN, GSI, DESY). Several astroparticle experiments (HiSCORE, CTA, IceCube, LHAASO, km3Net, IceCube-Gen2) [2,3] have also decided on the use of hardware based on the common and open WR solution. Non-White Rabbit devices may be connected to the WR network via a standard network interface card and operated within the WR network.

\section{White Rabbit facility}

The Roma Tor Vergata group is preparing a timing synchronization test facility based on the White Rabbit system. To fulfil the Cherenkov Telescope Array (CTA) requirements on event time stamping and dead time monitoring accuracy, the White Rabbit system has been chosen by the CTA Consortium. This facility will be used for the next gamma array telescope Cherenkov Telescope Array (CTA) $[4,5]$ but also for other future experiments. A common requirement for all the telescopes is the time stamping of the measured events. The required relative accuracy of these time stamps for CTA is $<$ $10 \mathrm{~ns}$, the goal accuracy is $<2 \mathrm{~ns}$. This relative accuracy and precision must be achieved between all different types of telescopes and camera combinations.

Figure 2 a shows several PCs for mounting the SPECs $2 \mathrm{~b}$. The baseline ingredients are (1) WRSwitches [6] (WRS) ( Fig. 2 (c)) and (2) WR-Nodes (Fig. 2 (b)), connected by standard Gigabit Ethernet fibers. The WRS are arranged like in a normal ethernet-network; the central WRS (Grand Master Switch) acts as the time source (e.g. connected to a GPS antenna). White Rabbit is build on Gigabit Ethernet (1000base-BX10) and takes advantage of the Ethernet standards SyncE and Precision Time Protocol. It offers sub-ns precision, with excellent clock phase stability. It utilizes one fiber for each WR-node for both synchronization and user data, and compensates dynamically for clock drifts due to e.g. environmental influences (temperature).

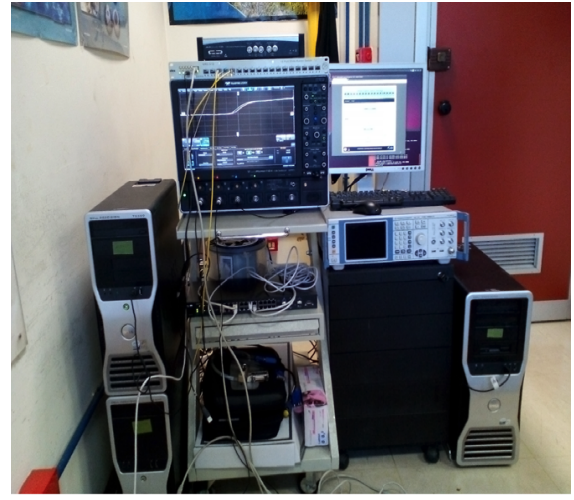

(a) WR setup

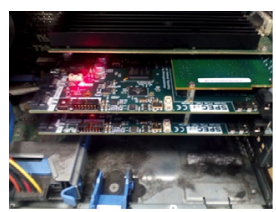

(b) WR SPEC cards

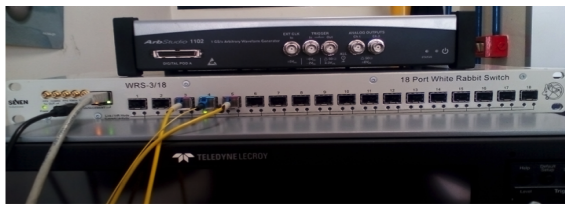

(c) WR switch

Figure 2: WR facility

The WR-node allows to interface the user system (eg. the DAQ of a detector station or a telescope) to the WR-time system: by either time-stamping signals from the detector and/or by supplying clockinformation (like PPS or periodic clock signals) to the detector. As the WR-node device we use for this work the Simple PCIe FMC carrier (SPEC) [7], shown in Fig. 2 (b) - a reliable workhorse of the 
WR- community. It has a Spartan-6 FPGA (with the WR PTP Core, optional custom firmware and software) and can accommodate FMC-mezzanine cards. We use the FMC-DIO5Ch [8], a 5 channel digital I/O card, for analog/digital trigger input, control signals, and PPS/MHz clock output (e.g. for clock performance tests).

\section{Results}

The calibration of the WR setup (optical fibers, internal delays), and the monitoring their stability are key measurements to ensure a good performance. In figure 3 we study the delay between the PPS from master and the PPS from slave when different optical fiber's length are used. The measurements were done using an oscilloscope to visualize both PPS. We observed that the fluctuations are well below the nanosecond. Also, we study the stability of the system for a period of 21 hours. In the same figure we show the delay as a function of time, where we can check if the delay between the PPS is always below the nanosecond.
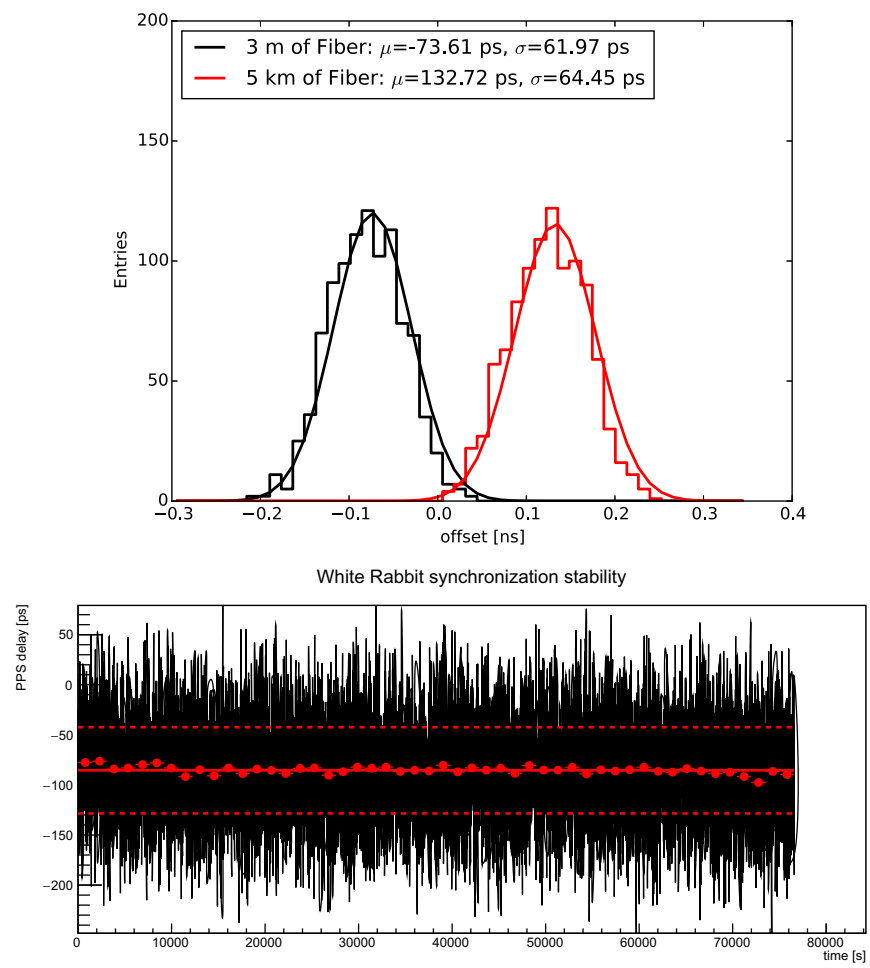

Figure 3: WR monitoring examples. Top figure shows the delay distribution between PPS from master and PPS from slave for two fibers of $3 \mathrm{~m}$ and $5 \mathrm{~km}$. Bottom figure shows the stability of the synchronization for a 21 hours run, red dots are the averages over 25 minutes, dashed lines indicates 1 sigma deviation. 


\section{Conclusions}

We have a multi purpose White Rabbit test facility at Tor Vergata Rome where the monitoring and the calibration of the system its done automatically using python scripts. In order to fulfill the CTA specification we have modify the White Rabbit firmware and implement the interface with the camera electronics. We also plan to implement the White Rabbit system providing the time synchronization for the camera demonstrator od ASTRI telescope [9].

\section{References}

[1] J. Serrano et al. The White Rabbit Project, ICALEPCS 2009, http://www.ohwr.org/projects/whiterabbit/wiki/

[2] M. Brückner et al., Time Synchronization with White Rabbit ? Experience from TunkaHiSCORE, ICRC2015, Proceedings of the $34^{\text {th }}$ ICRC 2015, The Hague, Netherlands, Proceedings of Science PoS (ICRC2015) 1041

[3] Oya, I. et al, arXiv:1509.01164, Proceedings of the $34^{\text {th }}$ ICRC 2015, The Hague, Netherlands, Proceedings of Science PoS (ICRC2015) 975

[4] http://cta-observatory.org

[5] WR-provider, www.sevensols.com

[6] http://www.ohwr.org/projects/white-rabbit/wiki/Switch

[7] http://www.ohwr.org/projects/white-rabbit/spec/wiki

[8] http://www.ohwr.org/projects/white-rabbit/fmc-dio-5chttla/wiki

[9] Pareschi G. et al. (2015), proc. TAUP 2015 